\title{
On the Extremal Steiner Wiener Index of Unicyclic Graphs
}

\author{
Yinqin Fan, Biao Zhao* \\ College of Mathematics and Systems Science, Xinjiang University, \\ Urumqi, Xinjiang 830017, P. R. China \\ fan_yqin@163.com, zhb_xj@163.com
}

(Received December 29, 2021)

\begin{abstract}
The Steiner $k$-Wiener index $S W_{k}(G)$ of a connected graph $G$ is defined as $S W_{k}(G)=\sum_{\substack{S \subseteq V(G) \\|S|=k}} d(S)$, where the $d(S)$ is equal to the subtree minimum size among subtrees of $G$ that connect $S$. A unicyclic graph is a connected graph with the same number of edges and vertices. In this paper, we study the lower and upper bounds of Steiner $k$-Wiener index of unicyclic graphs. In addition, we also obtain the second largest Steiner $k$-Wiener index among all trees.
\end{abstract}

\section{Introduction}

All graphs in this paper are assumed to be simple, finite and undirected. We refer to [1] for graph theoretical notation and terminology not explained here. For a connected graph $G$ of order at least two, and a set $S \subseteq V(G)$ with $S$ nonempty, a Steiner tree connecting $S$ or an $S$ Steiner tree (or simply, an $S$-tree) is a subgraph $T$ of $G$ that is a tree with $S \subseteq V(T)$. Let $G$ be a connected graph of order at least 2 , and $S$ be a nonempty set of vertices of $G$. Then the Steiner distance $d(S)$ is equal to

*Corresponding author. 
the subtree minimum size among subtrees of $G$ that connect $S$. Obviously, if $|S|=k, d(S) \geq k-1$. The determination of a Steiner tree in a graph is a discrete simulation of the well-known geometric Steiner problem, and Steiner trees are also used in multiprocessor computer networks. For more details on Steiner distance, we refer to $[2-5,13]$.

Topological index is a kind of mathematical invariants derived from structure diagram of compounds, which is often used to describe the physical, chemical and pharmacological characteristics of organic compounds. The Steiner $k$-Wiener index and Wiener index are two important topological indices. They are useful tools for studying the structural relationship of organic compounds in chemical research. The Steiner $k$-Wiener index $S W_{k}(G)$ of a connected graph $G$, proposed a generalization by Li et al [8], is defined by

$$
S W_{k}(G)=\sum_{\substack{S \subseteq V(G) \\|S|=k}} d(S) .
$$

The above definition implies $S W_{1}(G)=0$, and $S W_{n}(G)=n-1$ for a connected graph $G$ with $n$ vertices. For $k=2$, the Steiner 2-Wiener index $S W_{2}(G)$ coincides with the ordinary Wiener index.

In 2016, Li et al. [8] obtained the lower and upper bounds on Steiner $k$-Wiener index for connected graph $G$ and tree $T$, that is,

$$
\left(\begin{array}{l}
n \\
k
\end{array}\right)(k-1) \leq S W_{k}(G) \leq(k-1)\left(\begin{array}{l}
n+1 \\
k+1
\end{array}\right)
$$

for $2 \leq k \leq n-1$, the equality on the right if and only if $G$ is a path.

$$
\left(\begin{array}{l}
n-1 \\
k-1
\end{array}\right)(n-1) \leq S W_{k}(T) \leq(k-1)\left(\begin{array}{l}
n+1 \\
k+1
\end{array}\right)
$$

for $2 \leq k \leq n-1$, the star $S_{n}$ and the path $P_{n}$ attain the lower and upper bounds, respectively. And in [11], Li et al. determined the Steiner $k$-Wiener indices of cycle and wheel. For more studies on Steiner $k$-Wiener index, we refer to reader [6] and [8-10,12,14,15,17].

Let $U C(n)$ be the family of unicyclic graphs (i.e., connected graphs containing exactly one cycle) with $n$ vertices. Let $G$ be a unicyclic graph 
(of order $n$ ) with its unique cycle $C_{t}$ of length $t$, then $G-E\left(C_{t}\right)$ is a forest. $T_{1}, T_{2}, \cdots, T_{c}(0 \leq c \leq t)$ are all nontrivial trees of $G-E\left(C_{t}\right)$, then $\left|V\left(T_{i}\right)\right|=t_{i} \geq 2, n=t-c+\sum_{i=1}^{c} t_{i}$, and the common vertex of $C_{t}$ and $T_{i}$ is the cut vertex of $G, i=1,2, \cdots, c$. Such a unicyclic graph is denoted by $C_{t}\left(T_{t_{1}}, T_{t_{2}}, \cdots, T_{t_{c}}\right)$. If $c=0$, then $G=C_{n}$. In particular, for unicyclic graph $C_{t}\left(S_{t_{1}}, S_{t_{2}}, \cdots, S_{t_{c}}\right), S_{t_{i}}$ is a star with $t_{i}$ vertices, and the common vertex of $C_{t}$ and $S_{t_{i}}$ is the centre of $S_{t_{i}}$. And for unicyclic graph $C_{t}\left(P_{t_{1}}, P_{t_{2}}, \cdots, P_{t_{c}}\right), P_{t_{i}}$ is a path with $t_{i}$ vertices, and the common vertex of $C_{t}$ and $P_{t_{i}}$ is a pendent vertex of $P_{t_{i}}$. When $t=3, c=1, C_{3}\left(S_{n-2}\right)$ and $C_{3}\left(P_{n-2}\right)$ are shown in Figure 1.

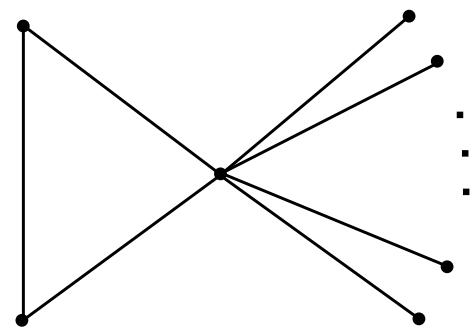

(1)

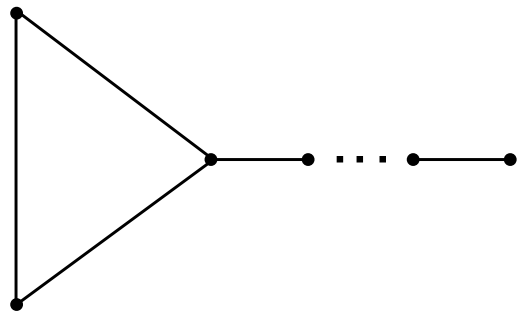

$(2)$

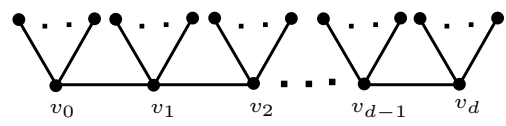

(3)

Figure 1. (1) $C_{3}\left(S_{n-2}\right)$; (2) $C_{3}\left(P_{n-2}\right)$; (3) $T_{d}^{v_{0}, \cdots, v_{d}}\left(m_{0}, \cdots, m_{d}\right)$.

The caterpillar tree, denoted by $T_{d}^{v_{0}, \cdots, v_{d}}\left(m_{0}, \cdots, m_{d}\right)$, is the tree obtained from $P_{d}=v_{0} v_{1} \cdots v_{d}$ by attaching $m_{i} \geq 0$ new vertices to $v_{i}$ for $0 \leq i \leq d$ (see Figure 1). For a set $S \subseteq V\left(C_{t}\left(S_{t_{1}}, S_{t_{2}}, \cdots, S_{t_{c}}\right)\right)$, the Steiner tree connecting $S$ of $C_{t}\left(S_{t_{1}}, S_{t_{2}}, \cdots, S_{t_{c}}\right)$ are caterpillar trees. 
Lemma 1.1. ([16]) Let $G$ be a unicyclic graph of order $n$, then

$$
S W_{2}\left(C_{3}\left(S_{n-2}\right)\right) \leq S W_{2}(G) \leq S W_{2}\left(C_{3}\left(P_{n-2}\right)\right)
$$

with the equality on the left (or on the right) if and only if $G \cong C_{3}\left(S_{n-2}\right)$ (or $G \cong C_{3}\left(P_{n-2}\right)$ ).

Lemma 1.2. ([r]) Let $G$ be a unicyclic graph of order $n$, and $t_{i} \geq 1$, then

$$
S W_{n-1}\left(C_{n}\right) \leq S W_{n-1}(G) \leq S W_{n-1}\left(C_{3}\left(P_{t_{1}}, P_{t_{2}}, P_{t_{3}}\right)\right),
$$

with the equality on the left (or on the right) if and only if $G \cong C_{n}$ (or $\left.G \cong C_{3}\left(P_{t_{1}}, P_{t_{2}}, P_{t_{3}}\right)\right)$.

In above Lemmas, the lower and upper bounds of the Steiner $k$-Wiener index of unicyclic graphs $U C(n)$ are determined for $k=2$ and $k=n-1$. We consider the lower and upper bounds of the Steiner $k$-Wiener index of $U C(n)$ for $3 \leq k \leq n-2$ in this paper. By a simple calculation, we can obtain the Steiner $k$-Wiener index of $U C(n)$ for $n \leq 5$, as shown in Table 1 .

\begin{tabular}{|l||l|l|l|}
\hline$U C(n)$ & $S W_{2}$ & $S W_{3}$ & $S W_{4}$ \\
\hline$C_{3}$ & 6 & & \\
$C_{4}$ & 8 & 8 & \\
$C_{3}\left(S_{2}\right)$ & 8 & 9 & \\
$C_{5}$ & 15 & 25 & 15 \\
$C_{4}\left(S_{2}\right)$ & 16 & 24 & 16 \\
$C_{3}\left(S_{3}\right)$ & 15 & 24 & 16 \\
$C_{3}\left(S_{2}, S_{2}\right)$ & 16 & 26 & 17 \\
$C_{3}\left(P_{3}\right)$ & 17 & 27 & 17 \\
\hline
\end{tabular}

Table 1. The Steiner $k$-Wiener index of $U C(n)$ for $n \leq 5$.

In this paper, we introduce some transformations for connected graphs (of order $n$ ) that do change their Steiner $k$-Wiener index for $3 \leq k \leq n-2$. Using these transformations, we study the lower bound of Steiner $k$-Wiener index of unicyclic graphs, and obtain the corresponding extremal graph as well. By studying the second largest Steiner $k$-Wiener index among all trees, we obtain the upper bound of Steiner $k$-Wiener index of unicyclic graphs. Keep in mind that we assume $n \geq 6$ for $U C(n)$. 


\section{The lower bound of Steiner $k$-Wiener in- dex of unicyclic graphs}

Let $G, H$ be two nontrivial connected graphs with $u \in V(G)$, and $v \in V(H)$. Let $G u H$ be the graph obtained from $G$ and $H$ by identifying $u$ with $v$.

Lemma 2.1. Let $G$ be a nontrivial connected graph with $u \in V(G), T_{m}$ be a nontrivial tree (of order $m$ ) with $v \in V\left(T_{m}\right)$. Let $G u S_{m}$ obtained from GuT $T_{m}$ by deleting the edges of $T_{m}$ and connect the vertices of $T_{m} \backslash\{u(v)\}$ to $u(v)$. For $k$ be an integer with $2 \leq k \leq n-1$, then

$$
S W_{k}\left(G u T_{m}\right) \geq S W_{k}\left(G u S_{m}\right),
$$

with the equality if and only if $G u T_{m} \cong G u S_{m}$.

Proof. For any set $S \subseteq V\left(G u T_{m}\right)=V\left(G u S_{m}\right)$ with $|S|=k$. We consider the following.

If $S \subseteq V(G)$, then $d_{G u T_{m}}(S)=d_{G u S_{m}}(S)$.

If $S \subseteq V\left(T_{m}\right)$, when $k>m, S$ is not exist. $S$ contribute the same to $S W_{k}\left(T_{m}\right)$ (or $S W_{k}\left(S_{m}\right)$ ) and $S W_{k}\left(G u T_{m}\right.$ ) (or $S W_{k}\left(G u S_{m}\right)$ ). Since $S W_{k}\left(T_{m}\right) \geq S W_{k}\left(S_{m}\right)$ with the equality if and only if $T_{m} \cong S_{m}$, then $S$ contribute to $S W_{k}\left(G u T_{m}\right)$ not less than $S W_{k}\left(G u S_{m}\right)$.

If $S \cap V(G \backslash\{u(v)\}) \neq \emptyset$ and $S \cap V\left(T_{m} \backslash\{u(v)\}\right) \neq \emptyset$. Whether $u(v)$ is contained in $S$ or not, $u(v)$ must be contained in the Steiner trees connecting $S$ for $G u T_{m}$ and $G u S_{m}$. We divide the Steiner tree $T_{G u T_{m}}(S)$ into two subtrees $T_{G}$ and $T_{T_{m}}$, where $V\left(T_{T_{m}}\right) \subseteq V\left(T_{m}\right), V\left(T_{G}\right) \subseteq V(G)$, $V\left(T_{T_{m}}\right) \cap V\left(T_{G}\right)=\{u(v)\},\left|V\left(T_{G u T_{m}}(S)\right)\right|=\left|V\left(T_{G}\right)\right|+\left|V\left(T_{T_{m}}\right)\right|-1$ and $d_{G u T_{m}}(S)=d_{G u T_{m}}\left(V\left(T_{T_{m}}\right)\right)+d_{G u T_{m}}\left(V\left(T_{G}\right)\right)$. Similarly, we divide the Steiner tree $T_{G u S_{m}}(S)$ into two subtrees $T_{G}$ and $T_{S_{m}}$. Since $d_{G u T_{m}}\left(V\left(T_{T_{m}}\right)\right) \geq d_{G u S_{m}}\left(V\left(T_{S_{m}}\right)\right), d_{G u T_{m}}\left(V\left(T_{G}\right)\right)=d_{G u S_{m}}\left(V\left(T_{G}\right)\right)$, then $d_{G u T_{m}}(S) \geq d_{G u S_{m}}(S)$.

From what has been discussed above, we can draw our conclusion.

By repeating the operation in Lemma 2.1, we can have the following corollary. 
Corollary 2.1. Let $G=C_{t}\left(T_{t_{1}}, T_{t_{2}}, \cdots, T_{t_{c}}\right) \neq C_{n}$ in $U C(n)$, and $k$ an integer with $2 \leq k \leq n-1$, then

$$
S W_{k}(G) \geq S W_{k}\left(C_{t}\left(S_{t_{1}}, S_{t_{2}}, \cdots, S_{t_{c}}\right)\right)
$$

with equality if and only if $G \cong C_{t}\left(S_{t_{1}}, S_{t_{2}}, \cdots, S_{t_{c}}\right)$.

Lemma 2.2. Let $C_{t}\left(S_{t_{1}}, S_{t_{2}}, \cdots, S_{t_{c}}\right) \neq C_{n}$ in $U C(n)$, and $k$ an integer with $2 \leq k \leq n-1$, then

$$
S W_{k}\left(C_{t}\left(S_{t_{1}}, S_{t_{2}}, \cdots, S_{t_{c}}\right)\right) \geq S W_{k}\left(C_{3}\left(S_{n-2}\right)\right)
$$

with equality if and only if $C_{t}\left(S_{t_{1}}, S_{t_{2}}, \cdots, S_{t_{c}}\right) \cong C_{3}\left(S_{n-2}\right)$.

Proof. Let $G=C_{t}\left(S_{t_{1}}, \cdots, S_{t_{c}}\right)$, and let us denote vertex sets of $G$ and $C_{3}\left(S_{n-2}\right)$ as $V(G)=V\left(C_{3}\left(S_{n-2}\right)\right)=\left\{u_{0}, u_{1}, \cdots, u_{n-1}\right\}$, where $u_{0}$ is a cut vertex of $G$ and $u_{0}$ is a unique vertex of degree $n-1$ in $C_{3}\left(S_{n-2}\right)$. For any set $S \subseteq V(G)$ with $|S|=k$, Steiner tree $T_{G}(S)$ is a caterpillar tree. Let $T_{G}(S)=T_{d}^{v_{0}, \cdots, v_{d}}\left(m_{0}, \cdots, m_{d}\right)$, where $v_{0}, v_{1}, \cdots, v_{d} \in V\left(C_{t}\right)$.

If $u_{0} \in S$, then $d_{C_{3}\left(S_{n-2}\right)}(S)=k-1 \leq d_{G}(S)$.

If $u_{0} \notin S$, then $d_{C_{3}\left(S_{n-2}\right)}(S)=k$ and $d_{G}(S) \geq k-1$.

When $d_{G}(S)=k-1=d_{C_{3}\left(S_{n-2}\right)}(S)-1$, then $V\left(T_{G}(S)\right)=S$. Let $N\left(u_{0}\right)$ be the set of neighbors of $u_{0}$ in $G$. Define the vertex $w \in V\left(T_{G}(S)\right)$ such that the distance between $u_{0}$ and $w$ is the shortest in $G$, then $w \in$ $V\left(C_{t}\right)$, and $w=v_{0}$ or $w=v_{d}$. Since $u_{0}$ is a cut vertex of $G$, then there is a vertex $w_{0} \in N\left(u_{0}\right)$ such that $d_{G}\left(w_{0}\right)=1$ and $w_{0} \notin S$. Let $\left.S^{\prime}=(S \backslash\{w\}) \cup\left\{w_{0}\right\}\right)$, then $d_{G}\left(S^{\prime}\right) \geq k+1=d_{C_{3}\left(S_{n-2}\right)}\left(S^{\prime}\right)+1$. In other words, if there is an $S$ that $d_{G}(S)=d_{C_{3}\left(S_{n-2}\right)}(S)-1$, then there must be $S^{\prime}$ such that $d_{G}\left(S^{\prime}\right) \geq d_{C_{3}\left(S_{n-2}\right)}\left(S^{\prime}\right)+1$.

Then, we have that $S W_{k}(G) \geq S W_{k}\left(C_{3}\left(S_{n-2}\right)\right)$.

Observation 2.1. Let $\alpha$ and $k$ be two positive integers. If $k=1$ or $k \geq \alpha-1$, then $\left(\begin{array}{l}\alpha \\ k\end{array}\right)<\alpha+1$; if $2 \leq k \leq \alpha-2$, then $\left(\begin{array}{l}\alpha \\ k\end{array}\right)>\alpha+1$.

Lemma 2.3. Let $k$ be an integer with $3 \leq k \leq n-2$, then

$$
S W_{k}\left(C_{n}\right)>S W_{k}\left(C_{3}\left(S_{n-2}\right)\right) .
$$


Proof. For any set $S \subseteq V\left(C_{n}\right)$ with $|S|=k$. If $d_{C_{n}}(S)=k-1$, there are $n$ such subsets, contributing to $S W_{k}$ by $n \times(k-1)$. And if $d_{C_{n}}(S) \geq k$, the number of such $S$ would be $\left(\begin{array}{l}n \\ k\end{array}\right)-n$.

For any set $S \subseteq V\left(C_{3}\left(S_{n-2}\right)\right)$ with $|S|=k \geq 3$. If $d_{C_{3}\left(S_{n-2}\right)}(S)=$ $k-1$, there are $\left(\begin{array}{l}n-1 \\ k-1\end{array}\right)$ such subsets, contributing to $S W_{k}$ by $\left(\begin{array}{l}n-1 \\ k-1\end{array}\right) \times$ $(k-1)$. And if $d_{C_{3}\left(S_{n-2}\right)}(S)=k$, the number of such $S$ would be $\left(\begin{array}{c}n-1 \\ k\end{array}\right)$. It follows that

$$
\begin{aligned}
& S W_{k}\left(C_{n}\right)-S W_{k}\left(C_{3}\left(S_{n-2}\right)\right) \\
& =\sum_{S \subseteq V\left(C_{n}\right)} d_{C_{n}}(S)-\sum_{\substack{S \subseteq V\left(C_{3}\left(S_{n-2}\right)\right) \\
|S|=k}} d_{C_{3}\left(S_{n-2}\right)}(S) \\
& \geq\left[n(k-1)+\left(\left(\begin{array}{l}
n \\
k
\end{array}\right)-n\right) k\right]-\left[\left(\begin{array}{l}
n-1 \\
k-1
\end{array}\right)(k-1)+\left(\begin{array}{c}
n-1 \\
k
\end{array}\right) k\right] \\
& =\left[n-\left(\begin{array}{l}
n-1 \\
k-1
\end{array}\right)\right](k-1)+\left[\left(\begin{array}{l}
n-1 \\
k-1
\end{array}\right)-n\right] k \\
& =\left(\begin{array}{l}
n-1 \\
k-1
\end{array}\right)-n .
\end{aligned}
$$

By Observation 2.1, we have that $\left(\begin{array}{l}n-1 \\ k-1\end{array}\right)-n>0$ for $3 \leq k \leq n-2$.

Combining Corollary 2.1 and Lemma 2.2-3, we get our main result immediately.

Theorem 2.4. For $G \in U C(n)(n \geq 6)$, let $k$ be an integer with $3 \leq k \leq$ $n-2$, then

$$
S W_{k}(G) \geq S W_{k}\left(C_{3}\left(S_{n-2}\right)\right)=\left(\begin{array}{l}
n-1 \\
k-1
\end{array}\right)(n-1),
$$

with the equality holds if and only if $G \cong C_{3}\left(S_{n-2}\right)$. 


\section{The upper bound of Steiner $k$-Wiener in- dex of unicyclic graphs}

Let $G$ be a unicyclic graph (of order $n$ ) with its unique cycle $C_{t}$ of length $t(3 \leq t \leq n), T_{1}, T_{2}, \cdots, T_{t}$ are all spanning trees of $G$. For an integer $k$ with $2 \leq k \leq n-1$, any set $S \subseteq V(G)$ with $|S|=k$, by Proposition 3.2 of [8], we have that $S W_{k}(G) \leq S W_{k}\left(T_{i}\right), i=1,2, \cdots, t$. Then, $S W_{k}(G) \leq \min \left\{S W_{k}\left(T_{1}\right), S W_{k}\left(T_{2}\right), \cdots, S W_{k}\left(T_{t}\right)\right\}$. If $G$ is not a cycle, then $G$ has at least one spanning tree that is not a path. When $G \in U C(n) \backslash\left\{C_{n}\right\}$, then $S W_{k}(G)<S W_{k}\left(P_{n}\right)$. It is necessary to determine the second largest Steiner $k$-Wiener index for trees.

Let $\mathcal{T}_{l}$ denote the set of trees (of order $n$ ) with $l$ leaves. Let $P_{n_{1}}$, $P_{n_{2}}, P_{n_{3}}$ be three paths, pairwise disjoint paths. Define three paths $P_{n_{1}}=$ $x_{1} x_{2} \cdots x_{n_{1}}, P_{n_{2}}=y_{1} y_{2} \cdots y_{n_{2}}$ and $P_{n_{3}}=z_{1} z_{2} \cdots z_{n_{3}}$, where $\left|V\left(P_{n_{i}}\right)\right|=$ $n_{i} \geq 2$. Let $P_{n_{1}} x_{n_{1}} P_{n_{2}}$ be the graph obtained from $P_{n_{1}}$ and $P_{n_{2}}$ by identifying $x_{n_{1}}$ with $y_{n_{2}}$, and $T_{3}\left(n_{1}, n_{2}, n_{3}\right)$ be the graph obtained from $P_{n_{1}} x_{n_{1}} P_{n_{2}}$ and $P_{n_{3}}$ by identifying $x_{n_{1}}$ with $z_{n_{3}}$. Then, $T_{3}\left(n_{1}, n_{2}, n_{3}\right) \in \mathcal{T}_{3}$, and every graph in $\mathcal{T}_{3}$ can be obtained in this way.

In this section, we first prove that $S W_{k}\left(T_{3}(2,2, n-2)\right)$ reaches the second largest Steiner $k$-Wiener index among all trees. Then, we get the upper bound of the Steiner $k$-Wiener index for unicyclic graphs by computing $S W_{k}\left(T_{3}(2,2, n-2)\right.$ for $3 \leq k \leq n-2$.

Let $G_{0}$ is a connected graph with $v \in V\left(G_{0}\right)$, and $G$ be the graph (of order $n$ ) obtained from $G_{0}$ and $P_{n_{1}} x_{n_{1}} P_{n_{2}}$ by identifying $v$ with $x_{n_{1}}$. Then we construct a new graphs $\widetilde{G}=G-y_{n_{2}-1} y_{n_{2}}+y_{n_{2}-1} x_{1}$ from $G$.

Lemma 3.1. Let $G$ and $\widetilde{G}$ be the two graphs (of order $n$ ) above, and $k$ an integer with $2 \leq k \leq n-1$, then

$$
S W_{k}(G)<S W_{k}(\widetilde{G})
$$

Proof. Let $T=P_{n_{1}} x_{n_{1}} P_{n_{2}}$, for any set $S \subseteq V(G)=V(\widetilde{G})$ with $|S|=k$.

If $S \subseteq V\left(G_{0}\right)$, then $d_{G}(S)=d_{\widetilde{G}}(S)$.

If $S \subseteq V(T), S$ contribute the same to $S W_{k}(G)$ and $S W_{k}(\widetilde{G})$ (when $k>n_{1}+n_{2}-1, S$ is not exist). 
If $S \cap V\left(G_{0} \backslash\left\{x_{n_{1}}\right\}\right) \neq \emptyset$ and $S \cap V\left(T \backslash\left\{x_{n_{1}}\right\}\right) \neq \emptyset$, then $d_{G}(S) \leq d_{\tilde{G}}(S)$. Moreover, since $k \leq n-1$, there exists an $S^{\prime} \subseteq V(G)=V(\widetilde{G})$ with $\left|S^{\prime}\right|=k$ that contain $y_{1}$ but not $x_{1}$, then $d_{G}\left(S^{\prime}\right)<d_{\widetilde{G}}\left(S^{\prime}\right)$.

So, we have that $S W_{k}(G)<S W_{k}(\widetilde{G})$.

By repeating the operation in Lemma 3.1, we can see that the tree with the second largest Steiner $k$-Wiener index must be in $\mathcal{T}_{3}$.

Lemma 3.2. Let $p, q$ and $k$ be three positive integers such that $p<q-1$. If $2 \leq k \leq q$, then $\left(\begin{array}{l}p \\ k\end{array}\right)+\left(\begin{array}{l}q \\ k\end{array}\right)>\left(\begin{array}{c}p+1 \\ k\end{array}\right)+\left(\begin{array}{c}q-1 \\ k\end{array}\right)$. If $k \geq q+1$, then $\left(\begin{array}{l}p \\ k\end{array}\right)+\left(\begin{array}{l}q \\ k\end{array}\right)=\left(\begin{array}{c}p+1 \\ k\end{array}\right)+\left(\begin{array}{c}q-1 \\ k\end{array}\right)$.

Proof. It follows that

$$
\begin{aligned}
& {\left[\left(\begin{array}{l}
p \\
k
\end{array}\right)+\left(\begin{array}{l}
q \\
k
\end{array}\right)\right]-\left[\left(\begin{array}{c}
p+1 \\
k
\end{array}\right)+\left(\begin{array}{c}
q-1 \\
k
\end{array}\right)\right]} \\
& =\left[\left(\begin{array}{l}
q \\
k
\end{array}\right)-\left(\begin{array}{c}
q-1 \\
k
\end{array}\right)\right]-\left[\left(\begin{array}{c}
p+1 \\
k
\end{array}\right)-\left(\begin{array}{l}
p \\
k
\end{array}\right)\right] \\
& =\left(\begin{array}{l}
q-1 \\
k-1
\end{array}\right)-\left(\begin{array}{c}
p \\
k-1
\end{array}\right) .
\end{aligned}
$$

If $2 \leq k \leq q$ then $\left(\begin{array}{l}q-1 \\ k-1\end{array}\right)-\left(\begin{array}{c}p \\ k-1\end{array}\right)>0$.

If $k \geq q+1$ then $\left(\begin{array}{l}q-1 \\ k-1\end{array}\right)-\left(\begin{array}{c}p \\ k-1\end{array}\right)=0$.

Let $T$ be a tree of order $n$ and $e=x y \in E(T)$. Denote by

$$
\begin{aligned}
& N_{x}(e)=\{z \in V(T): d(z, x)<d(z, y)\} \\
& N_{y}(e)=\{z \in V(T): d(z, x)>d(z, y)\} .
\end{aligned}
$$

And denote the cardinality $\left|N_{x}(e)\right|=n_{x}(e),\left|N_{y}(e)\right|=n_{y}(e)$, respectively. By the definitions, we have $n=n_{x}(e)+n_{y}(e)$. Denote by $\lambda_{T}(e)=$ 
$\max \left\{n_{x}(e), n_{y}(e)\right\}$ and $\mu_{T}(e)=\min \left\{n_{x}(e), n_{y}(e)\right\}$. There is a formula for the Steiner $k$-Wiener index of a tree.

Lemma 3.3. ([12]) Let $T$ be a tree of order $n$, and $k$ an integer with $2 \leq k \leq n-1$. Then

$$
S W_{k}(T)=(n-1)\left(\begin{array}{l}
n \\
k
\end{array}\right)-\sum_{e \in E(T)}\left[\left(\begin{array}{c}
n_{x}(e) \\
k
\end{array}\right)+\left(\begin{array}{c}
n_{y}(e) \\
k
\end{array}\right)\right] .
$$

Let $T=T_{3}\left(n_{1}, n_{2}, n_{3}\right) \in \mathcal{T}_{3}$, define three paths $P_{n_{1}}=x_{1} x_{2} \cdots x_{n_{1}}$, $P_{n_{2}}=y_{1} y_{2} \cdots y_{n_{2}}$ and $P_{n_{3}}=z_{1} z_{2} \cdots z_{n_{3}}$, pairwise disjoint paths, where $\left|V\left(P_{i}\right)\right|=n_{i} \geq 2(i=1,2,3)$, and $n_{3}=\max \left\{n_{1}, n_{2}, n_{3}\right\}$. If one of $n_{1}$ and $n_{2}$ is greater than 2 , without loss of generality, suppose $n_{1}>2$ such that $\widehat{T}=T-x_{1} x_{2}+z_{1} x_{1}$ from $T$.

Lemma 3.4. Let $T$ and $\widehat{T}$ be the two graphs (of order $n$ ) above, then $S W_{k}(T)<S W_{k}(\widehat{T})$ for $2 \leq k<n_{1}+n_{2}$ and $S W_{k}(T)=S W_{k}(\widehat{T})$ for $n_{1}+n_{2} \leq k \leq n-1$.

Proof. Let $e_{i}, f_{j} \in E(T)$ such that $e_{i}=x_{i} x_{i+1}$ and $f_{j}=z_{j} z_{j+1}, i=$ $1,2, \cdots, n_{1}, j=1,2, \cdots, n_{3}$. For an arbitrary edge $e \in E(T)$, a feasible map from $T$ to $\widehat{T}$ is a bijection $\varphi: E(T) \rightarrow E(\widehat{T})$ such that:

(1) $\lambda_{T}(e)=\lambda_{\widehat{T}}(\varphi(e)), e \in E\left(P_{n_{2}}\right)$,

(2) $\lambda_{T}\left(e_{i}\right)=\lambda_{\widehat{T}}\left(\varphi\left(e_{i+1}\right)\right), e_{i} \in E\left(P_{n_{1}}\right)\left(1 \leq i \leq n_{1}-2\right)$,

(3) $\lambda_{T}\left(f_{j}\right)=\lambda_{\widehat{T}}\left(\varphi\left(f_{j-1}\right)\right), f_{j} \in E\left(P_{n_{3}}\right)\left(2 \leq j \leq n_{3}-1\right)$,

(4) $\lambda_{T}\left(f_{1}\right)=\lambda_{\widehat{T}}\left(\varphi\left(e_{1}\right)\right)$.

By Lemma 3.3, we have that

$$
\begin{aligned}
& S W_{k}(\widehat{T})-S W_{k}(T) \\
& =\left[(n-1)\left(\begin{array}{l}
n \\
k
\end{array}\right)-\sum_{e \in E(\widehat{T})}\left[\left(\begin{array}{c}
\lambda_{\widehat{T}}(e) \\
k
\end{array}\right)+\left(\begin{array}{c}
\mu_{\widehat{T}}(e) \\
k
\end{array}\right)\right]\right] \\
& -\left[(n-1)\left(\begin{array}{l}
n \\
k
\end{array}\right)-\sum_{e \in E(T)}\left[\left(\begin{array}{c}
\lambda_{T}(e) \\
k
\end{array}\right)+\left(\begin{array}{c}
\mu_{T}(e) \\
k
\end{array}\right)\right]\right]
\end{aligned}
$$




$$
\begin{aligned}
& =\sum_{e \in E(T)}\left[\left(\begin{array}{c}
\lambda_{T}(e) \\
k
\end{array}\right)+\left(\begin{array}{c}
\mu_{T}(e) \\
k
\end{array}\right)\right]-\sum_{e \in E(\widehat{T})}\left[\left(\begin{array}{c}
\lambda_{\widehat{T}}(e) \\
k
\end{array}\right)+\left(\begin{array}{c}
\mu_{\widehat{T}}(e) \\
k
\end{array}\right)\right] \\
& =\left[\left(\begin{array}{c}
\lambda_{T}\left(e_{n_{1}-1}\right) \\
k
\end{array}\right)+\left(\begin{array}{c}
\mu_{T}\left(e_{n_{1}-1}\right) \\
k
\end{array}\right)\right]-\left[\left(\begin{array}{c}
\lambda_{\widehat{T}}\left(\varphi\left(f_{n_{3}-1}\right)\right) \\
k
\end{array}\right)+\left(\begin{array}{c}
\mu_{\widehat{T}}\left(\varphi\left(f_{n_{3}-1}\right)\right) \\
k
\end{array}\right)\right] \\
& =\left[\left(\begin{array}{c}
n_{1}-1 \\
k
\end{array}\right)+\left(\begin{array}{c}
n_{2}+n_{3}-1 \\
k
\end{array}\right)\right]-\left[\left(\begin{array}{c}
n_{3} \\
k
\end{array}\right)+\left(\begin{array}{c}
n_{1}+n_{2}-2 \\
k
\end{array}\right)\right] .
\end{aligned}
$$

Since $n_{1}, n_{2} \leq n_{3}$, then $n_{1}-1<n_{2}+n_{3}-2$. Let $n_{3}=n_{1}+\beta, \beta \geq 0$, by Lemma 3.2, we have that

$$
\begin{aligned}
{\left[\left(\begin{array}{c}
n_{1}-1 \\
k
\end{array}\right)+\left(\begin{array}{c}
n_{2}+n_{3}-1 \\
k
\end{array}\right)\right] } & \geq\left[\left(\begin{array}{c}
n_{1} \\
k
\end{array}\right)+\left(\begin{array}{c}
n_{2}+n_{3}-2 \\
k
\end{array}\right)\right] \\
& \geq \cdots \\
& \geq\left[\left(\begin{array}{c}
n_{1}+\beta \\
k
\end{array}\right)+\left(\begin{array}{c}
n_{2}+n_{3}-2-\beta \\
k
\end{array}\right)\right] \\
& =\left[\left(\begin{array}{c}
n_{3} \\
k
\end{array}\right)+\left(\begin{array}{c}
n_{1}+n_{2}-2 \\
k
\end{array}\right)\right] .
\end{aligned}
$$

Thus, we have

$$
\left\{\begin{array}{lll}
S W_{k}(\widehat{T})-S W_{k}(T)>0 & \text { if } & 2 \leq k \leq n_{1}+n_{2}-1 \\
S W_{k}(\widehat{T})-S W_{k}(T)=0 & \text { if } \quad k \geq n_{1}+n_{2} .
\end{array}\right.
$$

By repeating the operation in Lemma 3.4, $S W_{k}\left(T_{3}(2,2, n-2)\right)$ gets the maximum Steiner $k$-Wiener index for all trees in $\mathcal{T}_{3}$. So, $S W_{k}\left(T_{3}(2,2, n-\right.$ $2)$ ) is the second largest Steiner $k$-Wiener index among all trees. Then, we can have the following Theorem.

Theorem 3.5. Let $T \neq P_{n}$ be a tree of order $n$, and $k$ an integer with $2 \leq k \leq n-1$, then

$$
S W_{k}(T) \leq S W_{k}\left(T_{3}(2,2, n-2)\right)<S W_{k}\left(P_{n}\right),
$$


where $S W_{k}\left(T_{3}(2,2, n-2)\right)=(k-1)\left(\begin{array}{l}n+1 \\ k+1\end{array}\right)-\left(\begin{array}{l}n-2 \\ k-1\end{array}\right)+\left(\begin{array}{c}1 \\ k-1\end{array}\right)$.

Proof. Let $T=T_{3}(2,2, n-2)$, define three paths $P_{2}=x_{1} x_{2}, P_{2}=y_{1} y_{2}$ and $P_{n-2}=z_{1} z_{2} \cdots z_{n-2}$, pairwise disjoint paths. By the formula (3.1) in Lemma 3.4, we have that $P_{n}=\widehat{T}=T-x_{1} x_{2}+z_{1} x_{1}$, and

$$
\begin{aligned}
& S W_{k}\left(P_{n}\right)-S W_{k}(T)=S W_{k}(\widehat{T})-S W_{k}(T) \\
& =\left[\left(\begin{array}{c}
n_{1}-1 \\
k
\end{array}\right)+\left(\begin{array}{c}
n_{2}+n_{3}-1 \\
k
\end{array}\right)\right]-\left[\left(\begin{array}{c}
n_{3} \\
k
\end{array}\right)+\left(\begin{array}{c}
n_{1}+n_{2}-2 \\
k
\end{array}\right)\right] \\
& =\left[\left(\begin{array}{l}
1 \\
k
\end{array}\right)+\left(\begin{array}{c}
n-1 \\
k
\end{array}\right)\right]-\left[\left(\begin{array}{l}
2 \\
k
\end{array}\right)+\left(\begin{array}{c}
n-2 \\
k
\end{array}\right)\right] \\
& =\left(\begin{array}{l}
n-2 \\
k-1
\end{array}\right)-\left(\begin{array}{c}
1 \\
k-1
\end{array}\right) .
\end{aligned}
$$

Then, $S W_{k}\left(T_{3}(2,2, n-2)\right)=S W_{k}\left(P_{n}\right)-\left(\begin{array}{l}n-2 \\ k-1\end{array}\right)+\left(\begin{array}{c}1 \\ k-1\end{array}\right)$.

If $G \in U C(n) \backslash\left\{C_{n}\right\}, T_{i}(i=1, \cdots, t)$ is a spanning tree of $G$, then $S W_{k}(G) \leq \min \left\{S W_{k}\left(T_{1}\right), S W_{k}\left(T_{2}\right), \cdots, S W_{k}\left(T_{t}\right)\right\} \leq S W_{k}\left(T_{3}(2,2, n-\right.$ $2)$ ). For any set $S \subseteq V\left(C_{3}\left(P_{n-2}\right)\right)=V\left(T_{3}(2,2, n-2)\right)$ with $|S|=k \geq 3$, it is easy to see that $d_{C_{3}\left(P_{n-2}\right)}(S)=d_{T_{3}(2,2, n-2)}(S)$. Then $S W_{k}\left(C_{3}\left(P_{n-2}\right)\right)=$ $S W_{k}\left(T_{3}(2,2, n-2)\right)$.

Now let's compare the Steiner $k$-Wiener indices of $C_{n}$ to $C_{3}\left(P_{n-2}\right)$.

Lemma 3.6. Let $k$ be an integer with $3 \leq k \leq n-2$, then

$$
S W_{k}\left(C_{n}\right)<S W_{k}\left(C_{3}\left(P_{n-2}\right)\right) .
$$

Proof. Let $C_{n}=a_{1} a_{2} \cdots a_{n} a_{1}$ and $V\left(C_{3}\left(P_{n-2}\right)\right)=\left\{b_{1}, b_{2}, \cdots, b_{n}\right\}$, the vertex $b_{1}$ is the pendent vertex of $C_{3}\left(P_{n-2}\right)$, and denote the path $P_{n-2}=$ $b_{1} b_{2} \cdots b_{n-2}$. For $a_{i} \in V\left(C_{n}\right)$, a feasible map from $C_{n}$ to $C_{3}\left(P_{n-2}\right)$ is a bijection $\psi: V\left(C_{n}\right) \longrightarrow V\left(C_{3}\left(P_{n-2}\right)\right)$ such that $\psi\left(a_{i}\right)=b_{i}, i=$ $1,2 \cdots, n$. For any set $S \subseteq V\left(C_{3}\left(P_{n-2}\right)\right)$ with $|S|=k$, it is easy to see that $d_{C_{3}\left(P_{n-2}\right)}(S) \geq d_{C_{n}}(S)$. Moreover, since $k \leq n-2$, there must be 
$S^{\prime} \subseteq V\left(C_{3}\left(P_{n-2}\right)\right)$ with $\left|S^{\prime}\right|=k$ such that $d_{C_{3}\left(P_{n-2}\right)}\left(S^{\prime}\right)>d_{C_{n}}\left(S^{\prime}\right)$. Then, we have that $S W_{k}\left(C_{3}\left(S_{n-2}\right)\right)>S W_{k}\left(C_{n}\right)$.

From what has been discussed above, we obtain the upper bound of Steiner $k$-Wiener index of unicyclic graphs.

Theorem 3.7. For $G \in U C(n)(n \geq 6)$, let $k$ be an integer with $3 \leq k \leq$ $n-2$, then

$$
S W_{k}(G) \leq S W_{k}\left(C_{3}\left(P_{n-2}\right)\right)=(k-1)\left(\begin{array}{l}
n+1 \\
k+1
\end{array}\right)-\left(\begin{array}{l}
n-2 \\
k-1
\end{array}\right) .
$$

Acknowledgment: This work is supported by the Natural Science Foundation of Xinjiang Province (No. 2021D01C069) and the National Natural Science Foundation of China (grant number 12161085).

\section{References}

[1] J. A. Bondy, U. S. R. Murty, Graph Theory, Springer, New York, 2008.

[2] J. Cáceres, A. Márquez, M. L. Puertas, Steiner distance and convexity in graphs, Eur. J. Comb. 29 (2008) 726-736.

[3] G. Chartrand, O. R. Oellermann, S. Tian, H. B. Zou, Steiner distance in graphs, Časopis Pest. Mat. 114 (1989) 399-410.

[4] P. Dankelmann, O. R. Oellermann, H. C. Swart, The average Steiner distance of a graph, J. Graph Theory 22 (1996) 15-22.

[5] P. Dankelmann, O. R. Oellermann, H. C. Swart, On the average Steiner distance of graphs with prescribed properties, Discr. Appl. Math. 79 (1997) 91-103.

[6] I. Gutman, B. Furtula, X. Li, Multicenter Wiener indices and their applications, J. Serb. Chem. Soc. 80 (2015) 1009-1017.

[7] J. Lai, M. Liu, The Steiner (n-1)-Wiener index of unicyclic graphs, J. Lanzhou Jiaotong Univ. 40 (2021) 141-143.

[8] X. Li, Y. Mao, I. Gutman, The Steiner Wiener index of a graph, Discuss. Math. Graph Theory 36 (2016) 455-465. 
[9] X. Li, Y. Mao, I. Gutman, Inverse problem on the Steiner Wiener index, Discuss. Math. Graph Theory 38 (2018) 83-95.

[10] Z. Li, B. Wu, The Steiner Wiener index of trees with given bipartition, MATCH Commun. Math. Comput. Chem. 86 (2021) 363-373.

[11] X. Li, Z. Zhang, Results on two kinds of Steiner distance-based indices for some classes of graphs, MATCH Commun. Math. Comput. Chem. 84 (2020) 567-578.

[12] L. Lu, Q. Huang, J. Hou, X. Chen, A sharp lower bound on the Steiner Wiener index for trees with given diameter, Discr. Math. 341 (2018) 723-731.

[13] Y. Mao, B. Furtula, Steiner distance in chemical graph theory, MATCH Commun. Math. Comput. Chem. 86 (2021) 211-287.

[14] Y. Mao, Z. Wang, I. Gutman, Steiner Wiener index of graph products, Trans. Comb. 5 (2016) 39-50.

[15] Y. Mao, Z. Wang, Y. Xiao, C. Ye, Steiner Wiener index and connectivity of graphs, Util. Math. 102 (2017) 51-57.

[16] Z. Tang, H. Deng, The $(n, n)$-graphs with the first three extremal Wiener index, J. Math. Chem. 43 (2008) 60-74.

[17] J. Zhang, G. Zhang, H. Wang, X, Zhang, Extremal trees with respect to the Steiner Wiener index, Discr. Math. Algor. Appl. 11 (2019) \#1950067. 